Even the best texts have shortcomings. I wish this book had better quality reproduction of neuroimaging. I also missed more discussion of controversies in pediatric neurology particularly of management issues. For example, whether one should anticoagulate infants or children with venous-sinus thrombosis and under what circumstances, or elaborate on the utility of corticosteroid treatment in bacterial meningitis in the $H$. influenza vaccine era. I would also like modern texts to use more evidence-based medicine principles to assess different treatment modalities, possibly by using a grading system of the methodological soundness of the referred studies.

Occasionally, important pieces of information are missing. For example, the fact that children with the Wolfe-Hirschhorn Syndrome almost always suffer from intractable epilepsy often similar to the epilepsy observed in the Angelman Syndrome, was not mentioned.

However, my criticism is minor compared with the achievements of the authors and editors. I placed this text in my library at a hand's reach next to Aicardi's and Volpe's texts and have been opening it almost daily to look up different issues.

In conclusion, here is an excellent text in Pediatric Neurology with an emphasis on basic science and the clinical picture of common and rare disorders and at a very affordable price - almost one quarter of the price of Aicardis'. I strongly recommend it to every clinician with an interest in Pediatric Neurology.

Gabriel M. Ronen Hamilton, Ontario

\section{Drug Treatment of Migraine and other Headaches Volume 17. Monograms in Clinical Neuroscience. 2000. Edited by H.C. Diener, Published by Karger. 372 pages. C $\$ 281.50$ approx.}

Introduction: In the preface, Diener writes about the need for a book (on headache) that concentrates on current drug treatment based on pharmacological knowledge and evidenced-based medicine. Most of the book (307 of 364 pages) is devoted to migraine - almost exclusively migraine with and without aura.

Comparison: I compared the monogram edited by Diener with two others: (i) "Headache", edited by Goadsby \& Silberstein (1997) and (ii) "The Headaches" 2nd edition, edited by Olesen et al (2000). All three are multi-authored. Several authors have contributed chapters to each of these monograms. Hence, much of the information is "triplicated".

Strengths: There is considerable pharmacological information on a number of drugs, especially the triptans, fulfilling one of Diener's objectives.

Evidenced-based?: Although all chapters list and discuss a number of studies and references, the authors do not tell us how these were chosen for the review. First, how did the authors "search" the literature? Second, if they "selected" the references they included, what were the criteria for including/excluding references? The authors do not use the commonly accepted (at least in North America) "Levels of Evidence" and "Strength of Recommendations" in presenting the results. The two-part review by Pryse-Phillips, et al CMAJ 1997;156:1273-87; and CMAJ 1998;159:47-54 could have served as a model.

\section{Limitations/Omissions:}

(i) The use of sumatriptan in pediatric migraine is mentioned (pp 103-104). Otherwise, headaches in children and adolescents are not dealt with. (ii) The chronic daily headache syndrome, met with in adults and children, is not discussed.

(iii)Benign exertional headache, another common headache syndrome encountered is also not mentioned.

(iv) Most of us (including those who practice pediatric neurology) have accepted the International Headache Society Classification and Criteria. Since these were first published in 1988, a number of peer reviewed papers, especially dealing with children, have discussed limitations of the original classification and criteria and made suggestions for revision. I am disappointed at the absence of a critical re-appraisal of this subject in the chapter on classification and symptoms (pp 16-23).

(v) I could not find any discussion on topiramate or botulinum toxin in the management of headache and these are not referenced in the index, suggesting that the review of drug treatment may not have been as current as one would have liked.

(vi) Non-pharmacologic and "alternative" treatments are not discussed. In the preface, Diener felt the inclusion of this topic would have been too much for one volume.

\section{Conclusions:}

(i) The monogram edited by Diener is not as inclusive as the larger $(1000+$ pages $)$ monogram edited by Olesen et al. For this reason, the latter is more likely to serve as a current reference for headaches (in adults). Readers should compare the two and select the one that best suits individual needs.

(ii) Neither has a chapter critically appraising the International Headache Society Classification and Criteria.

(iii)Neither does justice to headaches in children and adolescents.

\section{Shashi Seshia \\ Winnipeg, Manitoba}

(The) Keyhole Concept in Neurosurgery with EndoscopicAssisted Microsurgery and Case Studies. 1999. Edited by Axel Perneczky, Wibke Muller-Forell, Erik van Lindert, Georg Fries. Published by Thieme. 264 pages. C\$268.65 approx.

(The) Keyhole Concept in Neurosurgery provides an interesting and concise description of the neurosurgical equivalent of 'minimally invasive surgery'. The German authors include three neurosurgeons (AP, EvL, GF) and a neuroradiologist (WM-F). Consistent with Thieme publications, the layout is attractive and accessible. The book is organized into two main sections. The first part of the book is divided into seven chapters describing the rationale, history, technology, strategy and future of 'keyhole' neurosurgery. The second part describes 25 case studies illustrating the potential for this surgical approach for a number of neurosurgical pathologies.

The authors begin the text with a definition of 'minimally invasive-maximally effective'procedures in neurosurgery. They are careful to reassure the reader that a smaller opening is not necessarily a better opening if the pathology cannot be treated adequately. However, the inference is made that an opening of no larger than $2.5 \mathrm{~cm}$ is adequate to handle the majority of cranial diseases - from basilar tip aneurysms to clival meningiomas. The advantages of the 'keyhole' approach are described as time efficiency, improved wound healing, and diminished tissue trauma. At the same time, the pathologic tissue is managed with the same effectiveness as standard techniques. The second chapter provides 
an interesting discourse on the history of minimally invasive neurosurgery, which includes the evolution of diagnostic technology, neuroanatomy, instrumentation, hemostasis, microscopes and endoscopes. The following two chapters describe the concept of 'light and sight' which is effectively the combined advantages of the microscope with the endoscope. Chapter 5 is a largely ineffective description of the general neuroradiologic principles involved in imaging intracranial cerebrovascular and neoplastic disorders. The next chapter describes the technical considerations to be made in 'keyhole' approaches, which are not dramatically different from those required for any surgical approach to intracranial disease. Four standard 'keyhole' craniotomies are described in moderate detail, these include the supraorbital, subtemporal, interhemispheric and transcortical-transventricular approaches. The final chapter in this section muses on the future of 'keyhole' surgery, including the application of neuro-navigational systems, intra-operative imaging technology and robotics.

The book finishes with 25 illustrative cases. Each case includes a brief clinical summary, an overview of possible surgical approaches, the actual keyhole procedure employed, patient outcome and relevant comments. This section is replete with illustrations and photographs. Although the schematic drawings are effective and clear, many of the intra-operative pictures are murky and not entirely helpful. Included in the list of cases treated by these techniques are aneurysms (anterior and posterior circulation), meningiomas, pituitary adenomas, arterio-venous malformations, cavernomas, craniopharyngiomas and epidermoids. Operative complications are honestly reported.

The book stands as an 'interesting read'with the stated ambition of initiating interest and progress in the 'keyhole' concept. It does not specifically describe how the surgeons were able to work through such small openings or the actual advantages of involving an endoscope, outside of looking around corners. Although the concept is empirically sound, the book does not attempt to prove that the 'keyhole'approaches provide the equivalent patient outcome as standard craniotomies. Clearly the approach is feasible, but the reader is left with a desire to know the outcome of large series of patients treated in this fashion, the procedural time required while using keyhole openings and the learning curve for the approach. Nevertheless the text is a worthwhile investment for those who question what frontiers remain in neurosurgery and in what way they might be conquered.

Brian D. Toyota Vancouver, British Columbia

\section{Neuromuscular Diseases: From Basic Mechanisms to} Clinical Management. Monographs in Clinical Neurosciences: Vol. 18. 2000. Edited by F. Deymeer. Published by Karger. 196 pages. $C \$ 241.00$ approx.

This book is Volume 18 of a serial publication "Monographs in Clinical Neuroscience". This volume was edited by F. Deymeer of Istanbul, Turkey. It has 12 chapters, each one authored by one or more experienced neuromuscular clinicians in their respective fields. Because of multiple authorships in some chapters, a total of 25 authors contributed.

What type of readership did this book target? Clearly, mainly practicing neurologists, other specialists, and residents to serve as a concise reference source at the bedside. Although it is bigger than a pocket book-size reference, in principle, it could pass for one. The embraced subjects are both myopathies and diseases of the peripheral nervous system. However, one wonders what guided the editor to include or omit certain topics. For example, the omission of inflammatory myopathies, classical autoimmune myasthenia gravis and sporadic ALS is curious and represents a major deficiency. This particularly stands out as a paradox, since in a lead position, a 14 page chapter is devoted to myoblast transfer, a procedure that is not particularly promising for the treatment of muscle disease.

As with every multi-author publication, there is considerable variability of the caliber of individual chapters, both from the clinical and basic scientific standpoint. This reviewer finds Chapter $1,2,6,7$ and 12 to be particularly scholarly.

There are numerous larger, excellent, recent texts on myology as well as peripheral nervous system diseases on the market. This book may find a niche in this crowded field on account of being concise and covering muscle and nerve diseases under the same cover. The relatively high price will not help in this endeavor.

George Karpati Montreal, Quebec

Cognitive Neurorehabilitation. 1999. Edited by Donald T. Stuss, Gordon Winocur, Ian H. Robertson. Published by Cambridge University Press. 385 pages. C\$154.00 approx.

One of the major challenges in neuroscience is to restore function following brain damage. In this regard, the ability to restore, in whole or in part, cognitive function following brain damage, from neurodegenerative disorders such as Alzheimer's disease, traumatic brain injury or stroke, would represent a pinnacle in neuroscience endeavours. The notion that, once the brain is damaged it cannot be repaired, no longer holds. In an age of explosive growth in the area of neuroscientific research at molecular, cellular, whole organism, and societal levels, the publication of Cognitive Neurorehabilitation is timely.

In 1995, the Rottman Research Institute of the Baycrest Centre for Geriatric Care, Toronto, Canada, held its 5th annual conference entitled, "Cognitive Rehabilitation of Acute and Age Related Brain Disorders". At the urging of the attendees, the organizers embarked on a project to compile the proceedings of this conference into a book. The presenters were asked to provide a detailed and up-to-date chapter on the subject they presented. The result of this endeavour is a more comprehensive edited book rather than a mere compilation of conference proceedings.

This book has four sections and a total of 22 chapters. Each section starts with an overview prepared by one of the editors.

Part I deals with "Mechanisms of Principles of Recovery". The chapters include comprehensive reviews of subjects ranging from neuroplasticity, neurotransplantation, neuroimaging, compensation in neurorehabilitation, sex hormones in recovery, and psychosocial environment in cognitive rehabilitation.

Evidence is provided that the nervous system does have the capacity to regenerate in animal models. The field of neuroimaging is now posed to make a significant impact on the care of patients with brain dysfunction, due to recent advances in neuroimaging techniques.

Part II deals with "Pharmacological Approaches". In this section there are chapters that deal with various aspects of neuroprotection, particularly in traumatic brain injury. A chapter on pharmacological 